\title{
Personalized prices and uncertainty in monopsony*
}

\author{
Roberto Burguet ${ }^{\dagger}$ and József Sákovics ${ }^{\ddagger}$
}

August 23, 2019

\begin{abstract}
We analyze personalized pricing by a monopsonist facing a finite number of $e x$ ante identical, capacity constrained suppliers with privately known costs. When the distribution of costs is sufficiently smooth and regular, the buyer chooses to make the same offer to all suppliers, leading to a posted price. When demand is sufficiently concave (convex) this price is lower (higher) than the classical monopsony price. In the limit as the seller capacities tend to zero, we obtain the classical monopsony price. Therefore, our model provides a decentralized micro-foundation for monopsony.
\end{abstract}

*We gratefully acknowledge financial support from the ESRC (Transparency in Procurement, ES/N00776X/1). We also thank Simon Board, Carmen Matutes and seminar participants at the European University Institute, U. Edinburgh, and U. Padova for useful comments.

${ }^{\dagger}$ University of Central Florida

†The University of Edinburgh and Universitat de les Illes Balears 


\section{Introduction}

In this paper we put forward a new model of decentralized pricing in monopsony ${ }^{1}$ and identify "mild" conditions under which the buyer's optimal price vector is a common price offered to all (ex ante identical) suppliers, independently of their number. This lack of qualification is important, as in our model the buyer faces uncertainty about the realized supply for any given price vector. It is thus reasonable to think that even a risk-neutral buyer would want to manage this uncertainty by using a heterogeneous price vector.

The classical model of monopsony postulates a single buyer who faces a deterministic supply curve resulting from the aggregation of suppliers' (marginal) costs. It is wellknown that the optimal linear (posted) price equates the "mark-down" ${ }^{2}$ to the reciprocal of this supply's elasticity. Note that, even if the monopsonist could offer different prices to different - but indistinguishable - suppliers with independent, random costs, setting this common price would still be optimal as long as the suppliers were many, each able to supply only an infinitesimal fraction of the monopsonist's demand. This is so, because the Law of Large Numbers ensures a deterministic aggregate supply, so that the monopsonist knows with certainty the quantity purchased at each price vector. Thus, in the classic scenario, the assumption of posted prices is without loss of generality.

Instead of this idealized situation, we are interested in the more realistic scenario, where the monopsonist is uncertain about the total supply he receives for a given vector of price offers. Think of an agricultural firm buying the crop of smallholders, not knowing at which price each of them would prefer to consume her crop rather than sell it. ${ }^{3}$ To manage this uncertainty, there is a potential role for personalized price offers to ex-ante homogeneous suppliers. In addition, we also wish to restrict attention to a selling mechanism that is decentralized, in the sense that the monopsonist contract with each supplier is "negotiated" independently.

\footnotetext{
${ }^{1}$ Needless to say, the entire analysis goes through equally for a monopoly.

${ }^{2}$ This is the eqivalent of the Lerner index for monopsony, the difference between the buyer's valuation of the marginal unit and the price as a proportion of the price: $\frac{P(S(p))-p}{p}$.

${ }^{3}$ Or a dominant supemarket buying milk from farms. While an oligopsony, an interesting example is the market for freshly minted Assistant Professors: the department makes the offers but has only a vague idea how many will be accepted.
} 
To address the above, we study the optimal (static) personalized pricing policy for a monopsonist that faces a finite number of ex ante identical, unit-capacity suppliers of privately known costs. Thus, the monopsonist may offer personalized (take-it-or-leaveit) prices to each supplier, but he must commit to trade at those prices if accepted. That is, agreements/commitments are bilateral: the terms of trade with each supplier is independent of the terms of trade with other suppliers. As we discuss in the conclusions, this personalized-pricing procedure has proven to be particularly useful for modeling price competition between oligopsonists, where it leads to novel insights.

Restricting the monopsonist strategy set to personalized pricing is, of course, not without loss of generality. As argued by Bulow and Roberts (1989), in the optimal mechanism for a monopsonist with full commitment power he announces a demand curve and solicits ask prices by sellers. The resulting aggregate supply schedule together with the announced demand is used to establish the market clearing price at which all the suppliers with ask prices below it trade. ${ }^{4}$ In such an auction, the terms of trade between the monopsonist and any individual seller depend on the bids of the other suppliers. Putting it differently, for any given supplier, the buyer commits not only to the way in which he will use the information she reveals, but also to how he will use the information revealed by all the other suppliers. Such a mechanism could not be implemented, for example, by delegated bargaining, where the monopsonist employs agents to bargain independently with different subsets of suppliers. Also, to verify that the monopsonist has delivered according to his promises to a single supplier, one would have to check the prices offered by all suppliers. Such a verification procedure may be highly impractical, especially when the suppliers would not want to reveal their costs to competitors. To capture situations where some verification might be necessary - say, because of suspicion of corruption - but a full verification is unworkable, we investigate monopsony pricing when the buyer is not able/willing to commit to a centralized mechanism. ${ }^{5}$

\footnotetext{
${ }^{4}$ Of course, in order to reduce the sellers' information rents, the announced demand schedule is distorted relative to the monopsonist's true demand function.

${ }^{5}$ For empirical evidence of firms using second-best organizational form (and thus pricing) see, for example, Thomas (2011) and references therein. See also McElheran (2014) on delegation. For a theoretical overview of decentralization see Mookherjee (2006).
} 
Our main result is that, under "mild" conditions on the distribution function of costs, posted prices are constrained optimal. The condition we identify is a strengthening of the traditional "regularity" condition in problems of trade under asymmetric information, which requires that the virtual $\operatorname{cost}^{6}$ of an arbitrary supplier be increasing. Roughly, we need the increase in virtual cost to be higher than the density of the cost distribution. Additionally, we show that the range of possible costs is also relevant. When there is a gap between the lowest possible cost and the buyer's lowest marginal valuation, the monopsonist may prefer to make fewer (serious) offers than there are traders (and demand). If on the other hand, there is a gap between the highest possible cost and the highest marginal valuation, the monopsonist may prefer to make some offers that are surely accepted in addition to different offers at lower prices. Notably, however, if the slope condition is met, all interior prices (that is, prices that will be refused and accepted both with positive probability) are the same. It is only when the cost distribution of suppliers is particularly convex that we observe heterogeneous interior prices offered to homogeneous suppliers.

We also show that even though the buyer offers a posted price, this price can be lower or higher than the corresponding classical monopsony price (roughly depending on whether the demand function is concave or convex, respectively). The reason is that the monopsony price is determined by a point elasticity, while the personalized price is optimized by taking expectations over the aggregate uncertainty.

Finally, we establish our convergence result: as aggregate supply is broken up into more and more suppliers, the outcome of our mechanism converges to the textbook monopsony pricing against a continuous supply function and the conditions for posted prices to be optimal are eventually always satisfied. That is, our model is a micro-foundation of the "invisible hand" in monopsony.

Following our concluding remarks, in Appendix A, we introduce heterogeneity in cost distributions and show that, contrary to the classic result, it is not necessarily the case that the less elastic market is offered the lower price. We find conditions for the standard insight to prevail. Just as the ones for the optimality of posted prices, these conditions

\footnotetext{
${ }^{6}$ If costs are random draws from the distribution function $F(c)$, the virtual cost function is given by $c+F(c) / f(c)$ (c.f. Myerson, 1981).
} 
are a strenthening of the standard condition with a term related to the slope of the monopsonist's demand function.

\subsection{A brief review of the literature}

The literature on optimal trading mechanisms ${ }^{7}$ is not directly relevant, as our interest here is in a second best. Another strand of the literature makes pairwise comparisons between bargaining, auctions and posted prices. ${ }^{8}$ Again, this is very different from our approach, where we stay with the standard pricing mechanism and investigate the benefits of discrimination in a hitherto unexplored context. Let us discuss some of the papers that are more closely related to our proposed mechanism.

Riley and Zeckhauser (1983) consider a seller with commitment power who is visited by buyers in sequence until she sells her unit. They show that the optimal strategy is a common take-it-or-leave-it price. Of course, due to the sequential resolution (and the unique item on offer) the aggregate uncertainty is minimal in this model.

In our setting, and assuming regularity, Bulow and Roberts (1989) showed that the mathematical problem of setting the optimal monopsony price is the same as setting the optimal reserve price in an auction, independently of the number of bidders. In other words, the optimal auction for ex ante identical suppliers involves a (common) reserve price, which equals the optimal take-it-or-leave-it offer to a single supplier: the monopsony price. Since Bulow and Roberts allow for supply uncertainty and obtain uniform (reserve) prices, one might consider that posted prices continue to be optimal. We show that this is not necessarily the case. ${ }^{9}$ Kotowski (2018) shows the contrapositive: when the demand function is not regular, personalized reserve prices may be optimal.

\footnotetext{
${ }^{7}$ Harris and Raviv (1981) is the classical study of the best mechanism of a single price setter faced with asymmetric information.

${ }^{8}$ Notable early contributions are Bester (1993) and Wang (1993, 1995).

${ }^{9}$ We knew that one had to be careful with extending the equivalence between setting optimal prices and reserve prices beyond the environment of Bulow and Roberts (1989). Burguet and Sákovics (1999) show that identical competing sellers will not set reserve prices equal to marginal cost in their auctions despite what happens in Bertrand competition.
} 
Winter (2004) also obtains that offering different prices to identical agents is useful. However, in his case the principal is using (some of the) prices as a coordination device in a multiple-equilibrium scenario.

Alonso et al. (2008) also look at the possibility of decentralized organizational structure but they assume that the monopsonist is constrained to name a single price. Therefore, the issue that determines whether a centrally set price or delegation to one of the local managers is optimal is how local managers are willing to report their private information about demand. As it turns out, when they are expected to widely disagree, decentralization is optimal.

Chen and Ishida (2013) consider the benefits of personalized pricing in a dynamic context. They show that price discrimination can increase a seller's expected profit if she can commit to dynamic price schedules. Otherwise, the ability to price discriminate not only is useless but can even harm the seller.

Interestingly, the logic of calculating expected marginal valuations is reminiscent of the analysis of Martin and Pindyck (2015) of the benefit of averting one catastrophe of several impending ones.

Finally, observe that an alternative solution - to our insistence on bilateral commitment - of dealing with a principal who cannot commit on how to represent the preferences of other agents, is the approach of designing credible mechanisms where the principal does not cheat only if his incentives are set right, as in Li (2017) and Akbarpour and Li (2018).

\section{The set-up: personalized pricing}

Consider a market with $Q$ unit-supply sellers and a risk neutral monopsonist with - just as in the classical monopsony model - a continuous, ${ }^{10}$ weakly decreasing (inverse) demand function $V($.$) that we can normalize, so that V(0)=1$ and $V(Q)=0$. Trade is restricted

\footnotetext{
${ }^{10}$ To highlight the consequences of indivisibilities in our model, we assume - as the textbooks - that the underlying supply and demand are continuous. The consequences of discontinuities in the classical context are standard.
} 
to be in integer units leading to (marginal) willingness to pay $v_{l}=\int_{l-1}^{l} V(x) d x \in[0,1]$ for the $l^{\text {th }}$ unit of the homogeneous good, $l \in\{1,2, \ldots, Q\}$, with $v_{l} \geq v_{l+1} \cdot{ }^{11}$ Seller $i$ 's cost (reservation price) is $c_{i}$ and it is $i$ 's private information. From the monopsonist's - and the other sellers' - point of view, $c_{i}$ (Seller $i$ 's "type") is the realization of an independent random variable, with - strictly increasing and common knowledge - distribution function $F_{i}($.$) and (differentiable) density function f_{i}($.$) on \left[\underline{c}_{i}, \bar{c}_{i}\right]$, where $0 \leq \underline{c}_{i}<\bar{c}_{i}$. To retain

simplicity and focus, we assume that $F_{i}($.$) is regular: c_{i}+\frac{F_{i}\left(c_{i}\right)}{f_{i}\left(c_{i}\right)}$ is monotone increasing. ${ }^{12}$

We study monopsony pricing as implemented by a simultaneous personalized offer to each seller, with full commitment. Note that, since there is aggregate uncertainty, the buyer risks having to acquire too many (or too few) units: he cannot adjust the prices and quantities ex post. This is an additional ingredient to the usual trade-off under complete information, between paying a low price and increasing the amount bought, that the monopsonist needs to take into account.

For clarity's sake, we first consider ex ante identical sellers, where each seller's cost is independently drawn from the same $F($.$) . We will later relax the symmetry assumption$ (c.f. Appendix 6).

Before continuing with our analysis, we first specify the benchmark case of classical monopsony and relate it to our personalized pricing model.

\subsection{The benchmark: classical monopsony}

In the classical model, the buyer (with inverse demand $V(q)$ ) faces a differentiable, increasing (inverse) supply function $S(q)$. Our set-up reduces to the classical model if we remove the uncertainty about the costs, so that the total supply at price $p$ coincides with the expected quantity that the $Q$ suppliers are willing to sell at that price: $S^{-1}(p)=Q F(p)$.

\footnotetext{
${ }^{11}$ As we allow for $v_{i}=0$ and as the demand for a higher number of units than there are available sellers would never be satisfied, it is without loss of generality to assume that both maximum aggregate supply and maximum demand are $Q$ units.

${ }^{12}$ This is the assumption that ensures that the first-order conditions imply the second-order conditions in the standard auction design problem (c.f. Myerson, 1981).
} 
With this analogue, the optimal monopsony quantity, $q^{M}$, calculated by equating marginal valuation with marginal expenditure, would be the solution to

$$
V\left(q^{M}\right)=\frac{d q^{M} S\left(q^{M}\right)}{d q^{M}}=S\left(q^{M}\right)+q^{M} S^{\prime}\left(q^{M}\right) .
$$

The optimal monopsony price would then be $p^{M}=S\left(q^{M}\right)$, which gives us a translation of (1) into prices:

$$
V\left(S^{-1}\left(p^{M}\right)\right)=p^{M}+S^{-1}\left(p^{M}\right) S^{\prime}\left(S^{-1}\left(p^{M}\right)\right)=p^{M}+\frac{S^{-1}\left(p^{M}\right)}{\left(S^{-1}\right)^{\prime}\left(p^{M}\right)} .
$$

Substituting $Q F\left(p^{M}\right)$ for $S^{-1}\left(p^{M}\right)$, we obtain ${ }^{13}$

$$
p^{M}+\frac{F\left(p^{M}\right)}{f\left(p^{M}\right)}=V\left(Q F\left(p^{M}\right)\right) .
$$

That is, the classical monopsonist posts a price that equates his marginal valuation with what in our model is the virtual cost of an arbitrary seller. ${ }^{14}$

\section{Interior offers}

The prices offered by the monopsonist can be classified into three groups: i) those that will surely be accepted; ii) those that will never be accepted (non-serious offers); and iii) those that may or may not be accepted with positive probability. We start by characterizing the first two types and then make an assumption on the cost distribution that rules them out, leaving us with all prices being interior.

Let us start with the option of making surely accepted offers. For the monopsonist, these have the obvious advantage of reducing uncertainty on the extensive margin.

\footnotetext{
${ }^{13}$ Given regularity, this equation has a unique solution.

${ }^{14}$ Note that equation (3) can also be written as the equality between the inverse of supply elasticity and the markdown:

$$
\frac{V\left(Q F\left(p^{M}\right)\right)-p^{M}}{p^{M}}=\frac{1}{\varepsilon^{s}\left(p^{M}\right)},
$$
}

where

$$
\varepsilon^{s}\left(p^{M}\right)=\left(\frac{d[Q F(p)]}{d p} \frac{p}{Q F(p)}\right)^{-1}=\left(\frac{f(p)}{F(p)} p\right)^{-1} .
$$


The intuition here is reminiscent of the setting of a reserve price in a standard auction, where the lowest buyer valuation is much higher than the seller's. In that case, a sale for the lowest possible valuation is so valuable that the marginal gain in price does not compensate for risking to lose the sale, resulting in no reserve price set. In the procurement context, we have the same scenario: if a unit can be bought for certain for a price that is a fraction of its valuation by the buyer, the expected gain from a more aggressive price offer may not outweigh the expected loss from possibly not buying it. The key factor therefore is the expected valuation of the unit minus the highest possible cost. When this difference is sufficiently large, it is optimal to make an offer that cannot be refused.

The following example illustrates.

Example 1 Assume that $v_{1}=1, v_{2}=0.2$, there are only two sellers, and $F(x)=4 x$ with support $[0, .25]$. The best single price for the buyer is $p=5 / 26 \approx .192$ and corresponding profit $\pi=10 / 13 \approx .769$ (details on how this price is obtained are in Subsection 3.1). If instead, the buyer sets one price equal to .25 (which is accepted for certain) and the price of .1 for the other seller, his expected profit is $.75+.1 \frac{.1}{.25}=.79$. Note that the optimal monopsony price without uncertainty of costs, as in Subsection 2.1, would be .125, buying one unit and expecting a profit of .875 .

When $\underline{c}>0$, any serious offer entails a price bounded away from zero. That is, if the buyer makes a number of offers above the number of units for which he has positive willingness to pay, then he risks incurring a loss that is also bounded away from zero. As the expected value of the marginal unit is not bounded away from zero, it may be optimal to make serious offers to only some of the potential sellers. The following example illustrates this possibility.

Example 2 Assume that $v_{1}=1$ and $v_{2}=0$, and that there are only two sellers. Let $F(x)=\frac{x-\underline{c}}{1-\underline{c}}$ with support $[\underline{c}, 1]$. If only one serious offer is made then $\Pi^{1}(p)=(1-p) F(p)$, and the first-order condition is $1=F(p) / f(p)+p$. Substituting in for $F$, we obtain $p=\frac{1+\underline{c}}{2}$ and thus $\Pi^{1}=\frac{1-\underline{c}}{4}$. If two (equal) serious offers are made, then the expected profit is $\Pi^{2}(b)=1-(1-F(b))^{2}-2 F(b) b$, leading to the first-order condition $1-F(b)=$ 
$F(b) / f(b)+b$. Substituting in for $F$, we obtain $b=\frac{1+\underline{c}-\underline{c}^{2}}{3-2 \underline{c}}$ and thus $\Pi^{2}=\frac{(1-\underline{c})^{2}}{3-2 \underline{c}}$. It is straightforward to see that $\Pi^{1}>\Pi^{2}$ if (and only if) $\underline{c}>1 / 2$.

The above discussion indicates that having a cost distribution such that with positive probability i) the cost is at least as high as the highest marginal valuation and ii) the cost is no more than the lowest valuation should ensure that all offers are interior. This is indeed the case, as we show next.

Definition $1 F($.$) has full support when its support includes that of the demand (\underline{c}=0$, $\bar{c} \geq 1)$.

Lemma 1 When $F($.$) has full support, all price offers are interior: p_{i} \in(\underline{c}, \bar{c})=(0,1)$.

Proof. See Appendix B.

The optimality of committing to buy from all comers may be somewhat surprising. Consider, for example, the extreme case when the buyer is looking for a single unit (and so $v_{l}=0$ for all $l>1$ ) from a large number $Q$ of suppliers. The intuition for making a serious offer to each seller even in this situation is, nonetheless, simple. If the buyer did not make a serious offer to some seller then his profit made on her would be zero. On the other hand, as long as the expected marginal valuation of the unit offered by this seller conditional on the offers made to the other sellers - is positive, by making an offer below this value, the monopsonist would receive a positive expected net marginal payoff. The expected marginal valuation of that seller's unit could be zero only if the entire demand is satisfied with probability one with the offers to the other sellers. However, in equilibrium that cannot happen. It would entail making an offer of 1 to (at least) one seller, leading to non-positive profits (on that seller). As a result, the optimal policy for the monopsonist must include serious offers to all sellers.

In the remainder of the paper we assume that $F($.$) has full support.$ 


\subsection{Common interior offers}

The crucial question is whether personalized pricing in the presence of supply uncertainty will result in identical individual price offers. As we will argue, the answer is affirmative if the probability distribution of costs satisfies a condition that is stronger than regularity. In Section 3.3, we discuss what might happen when the assumption is not satisfied.

Unlike in the case of regularity, where the restriction on the allowable cost (i.e., supply) distribution is exogenous, in our definition the constraint depends on the demand function as well. ${ }^{15}$

Definition 2 The distribution of costs, $F($.$) , is super-regular relative to \left\{v_{l}\right\}_{l=1,2, \ldots, Q}$ if: ${ }^{16} c+\frac{F(c)}{f(c)}-F(c) \max _{l \in\{1,2, \ldots, Q\}}\left\{v_{l}-v_{l+1}\right\}$ is strictly increasing in $c \leq 1$.

The constraint on the slopes of the cost distribution and the demand is stronger than regularity as $-F(c)$ is strictly decreasing. Also, the condition is the stronger the smaller the number of suppliers, as $\max _{l \in\{1,2, \ldots, Q\}}\left\{v_{l}-v_{l+1}\right\}$ is strictly decreasing in $Q$ (i.e., the number of steps from $V(0)=1$ to $V(Q)=0$ ). As an example, consider the family of power function probability distributions, where $F(c)=c^{z}$ for $c \in[0,1]$, for some positive value of $z$. All members of this family are log-concave (i.e., regular). However, for $\max _{l \in\{1,2, \ldots, Q\}}\left\{v_{l}-v_{l+1}\right\}=\Delta, F($.$) is super-regular relative to \left\{v_{l}\right\}_{l}$ only when $z<\frac{1+\sqrt{1+4 \Delta}}{2 \Delta}$. This constraint is strictest, when $\Delta=1: z<\frac{1+\sqrt{5}}{2} \approx 1.618$ (recall that the uniform distribution corresponds to $z=1$ ). On the other hand, it is satisfied by any $z$ for sufficiently small $\Delta$. That is, when the demand function, $V($.$) , is smooth and the number$ of suppliers is sufficiently large.

Super-regularity ensures that the system of first-order conditions has a unique, uniform solution, making it suboptimal for the buyer to target different parts of the supply separately - à la third-degree price discrimination (e.g. in case of a multi-peaked supply

\footnotetext{
${ }^{15} \mathrm{It}$ is straightforward to strengthen the assumption to be independent of $V($.$) : just substitute 1$ for

$\max _{l \in\{1,2, \ldots, Q\}}\left\{v_{l}-v_{l+1}\right\}$
${ }^{16}$ We let $v_{Q+1}=0$
} 
density). For $l \in\{0,1, \ldots, Q-1\}$, let

$$
\chi_{l}(x)=\left(\begin{array}{c}
Q-1 \\
l
\end{array}\right) F(x)^{l}(1-F(x))^{Q-l-1}
$$

denote the probability that $l$ out of $Q-1$ (independent) draws from the distribution $F$ are below $x$.

Proposition 1 When the cost distribution has full support and is super-regular relative to the buyer's demand, the optimal personalized pricing strategy is a unique (posted) price $p^{D}$ satisfying

$$
p^{D}+\frac{F\left(p^{D}\right)}{f\left(p^{D}\right)}=\sum_{l=0}^{Q-1} \chi_{l}\left(p^{D}\right) v_{l+1} .
$$

Proof. See Appendix B.

In other words, under super-regularity, the buyer does not (ab)use his ability to price discriminate: he offers to buy at the same price from all sellers. Thus, even under (aggregate) uncertainty of supply, our model offers a well-founded, game-theoretic foundation for posted prices. This qualitative feature replicates what is an assumption in the classical monopsony model. It is of particular note that this is not a convergence result: the one price result holds for any number of sellers.

\subsection{Posted prices and uncertainty}

The fact that, under super-regularity, the buyer names the same price for all sellers does not imply that this price coincides with the classical monopsony price. The decentralized posted price, $p^{D}$ in (4), differs from the classical monopsony price, $p^{M}$ in (3), construed as the optimal posted price when supply is (deterministic and) smooth and equals $Q F(p)$. The left-hand side of (3) and (4), the marginal expenditure (or virtual cost), is common to both expressions. However, the optimal price in the classic monopsony problem equates this marginal expenditure to the (marginal) willingness to pay at the optimal quantity. On the contrary, the optimal monopsony price under uncertainty, $p^{D}$, equals that marginal expenditure to the expectation of the marginal willingness to pay. That is, 
$p^{M}$ depends only on the demand function evaluated at the optimal quantity (the trade-off that determines it is local), whereas $p^{D}$ in (4) depends on the entire demand function.

Not surprisingly, there is no general ranking of these prices. The following example illustrates.

Example 3 Assume that $F(x)=x$ in $[0,1]$ and there are three sellers. Consider the following family of demand functions: ${ }^{17} v_{1}=1, v_{2}=y$, and $v_{3}=0$. Using (3) it is straightforward to verify that for $y<2 / 3, p^{M}=1 / 3$. On the other hand, (4) becomes $2 p=(1-p)^{2}+2(1-p) p y$, leading to $p^{2}(1-2 y)-(4-2 y) p+1=0$. For $y>.5$ this leads to $p^{D}>1 / 3$, and for $y<.5$ it leads to $p^{D}<1 / 3$.

Note that in the above example the threshold value of $y=.5$ corresponds to $V($. being "linear". This is not a coincidence. We can show that the intuition approximately holds in general. ${ }^{18}$

Proposition 2 If $V($.$) is sufficiently concave (convex) then p^{M} \geq(\leq) p^{D}$.

\section{Proof. See Appendix B.}

In other words, when the cost distribution has full support and is super-regular, and the demand function is sufficiently concave, the Bulow-Roberts intuition ${ }^{19}$ holds in our model: the price under uncertainty is lower than in the classical model. However, when either of these conditions is violated, the situation can change: we can have multiple prices and/or the price(s) offered can exceed $p^{M}$ (c.f. Example 3).

\footnotetext{
${ }^{17}$ For simplicity we work with discontinuous demand functions, it is trivial to see that the results would hold with arbitrarily close continuous approximations.

${ }^{18}$ The proof of Proposition 2 makes use of Jensen's inequality. Indeed, if it weren't for the fact that we are comparing a continuous function and a discrete function, Jensen's inequality would be all there would be behind that proposition.

${ }^{19}$ Recall that they say that the optimal reserve price in the procurement auction is same as the monopsony price and thus the actual price resulting from competitive bidding is (weakly) lower.
} 


\subsection{Prescinding from super-regularity}

While super-regularity is a reasonable assumption, it is clearly not always satisfied, in particular when the number of suppliers is not large. It is therefore pertinent to investigate the consequences of the failure of its components. In Subsection 4 we will show that, nonetheless, all the complications that might result from the failure of super-regularity disappear in the limit as the number of suppliers gets large (and their capacities decrease to zero). Indeed, for any $V($.$) , super-regularity will be satisfied by all cost distributions$ when the number of sellers is sufficiently large.

When super-regularity fails, the monopsonist may optimally price-discriminate between otherwise symmetric sellers, even if "classical" regularity is maintained. Note that this discrimination is different from third-degree price discrimination in that all suppliers are still assumed ex ante identical. That is, the endogenous price discrimination does not depend on any exogenous characteristic of suppliers.

The expected value of the marginal $l$ th unit that the buyer acquires increases by the step size multiplied by the probability of trade with the last inframarginal trader. This needs to be factored into the "regularity" of the virtual cost. When $v_{l}$ may be significantly larger than $v_{l+1}$, then given that a high offer is made to a seller, and so the probability that the $l$ th unit is acquired is high, the optimal offer to another seller may be low, and vice versa: if the offer made to the former is low, the best offer to the latter may be high. At the same time, super-regularity is far from necessary. The following example(s) illustrate:

Example 4 Supose that $v_{1}=1$ and $v_{2}=0$, and that there are only two (identical) sellers. consider $F(x)=x^{n}$ for $x \in[0,1]$ and $n \in \mathbb{N}$. Note that $\frac{d(x+F(x) / f(x))}{d x}=1+1 / n>0$ but $\frac{d(x+F(x) / f(x)-F(x))}{d x}=1+1 / n-n x^{n-1}<0$ for $x>\left(n^{-1}+n^{-2}\right)^{1 /(n-1)}<1$ and $n>1$. With the help of Mathematica it is immediate to see that for $n<6$ there are only symmetric positive real solutions to the system of first-order conditions (despite the cost function not being super-regular for any $n>1$ ). However for $n=6$ (and higher) there are three positive real solutions to the system of first-order conditions. A symmetric one with $p=.728752$ and two asymmetric ones with $p_{i}=.813675$ and $p_{j}=.608395$. Substituting them into 
the objective function, the first leads to an expected buyer profit of .0588231, while the latter(s) to .0592148. Thus, the optimal price vector is asymmetric.

\section{Large markets and convergence}

One of the goals of this paper is to provide a micro-foundation for the classical monopsony model that relies on the buyer being able to make personal commitments to individual suppliers, but without having the ability to make these commitments contingent on dealings with other sellers. Thus, we now show that indeed, in the limit, where the buyer faces a large number of (capacity constrained) small suppliers, his optimal price vector reduces to classical monopsony pricing (c.f. Section 2.1) without any additional assumption. That is, without any strengthening of regularity needed, and also without the need for full support.

Let's fix an integer $t$ and let $\delta=\frac{1}{t}$. Also, to save in notation without losing any generality, let $Q=1$. Suppose that each seller has an indivisible supply of $\delta$ units to sell with probability $\alpha$, and let $s_{i}$ be the per-unit price the monopsonist offers supplier $i$ for her supply. When $t=1$, this is the model analyzed in the previous section, for $Q=1$. As $t$ gets large, both the demand and the supply become a closer approximation of the underlying continuous functions $(V($.$) and F()):. \frac{v_{q}}{\delta}=\int_{q-\delta}^{q} \frac{1}{\delta} V(x) d x \rightarrow_{t \rightarrow \infty} V(q)$ and the realization of $t$ draws from $F($.$) converges to t F($.$) a.s. (by the Strong Law of$ Large Numbers). In other words, as $t$ increases without bound, our set-up converges to the classical monopsony set-up. The question is whether our predictions converge as well.

The answer is affirmative, and we will show it in two steps. First we will show that, under symmetric pricing, (4) converges to (3). Next, we will argue that the optimal solution to (4) near the limit must be symmetric, that is, a posted price, as long as $F($. is regular (which eventually means also super-regular).

Lemma 2 For any posted-price $p \in(0,1), \sum_{j=1}^{t} v_{j} \chi_{j-1}(p) \rightarrow_{t \rightarrow \infty} V(F(p))$.

Proof. See Appendix B. 
Lemma 3 For $t$ sufficiently large, the optimal personalized pricing scheme is a posted price.

Proof. See Appendix B.

Putting these two lemmas together, we have proved our main convergence result:

Proposition 3 For $t$ sufficiently large, the buyer-optimal personalized price vector converges to the classical monopsonist's posted price.

The assumption that the underlying (inverse) demand $V($.$) function is continuous$ greatly simplifies the proof of these convergence results. We conjecture that it is possible to extend the argument to an exogenously discontinuous demand, and to demonstrate that our convergence results do not hinge on the continuity of $V($.$) .$

\section{Conclusion}

In this paper, we delve into the micro-structure of monopsony and provide a "decentralized" mechanism, whose limit is the standard model. We show that there is no need for an "invisible hand": under mild conditions, optimal pricing with personalized commitment leads to a posted price even far away from the limit.

For simplicity, we have restricted attention to ex-ante identical sellers. The analysis can be straightforwardly extended to multiple types of sellers - that is, ex-ante asymmetric sellers - and our results are robust to such a generalization. In fact, as we also show in Appendix A, further insights arise: the standard result, that in multi-market monopsony the price in the market with more elastic supply is higher, need not hold. Once more, what guarantees that ranking of prices is not the relative size of the reverse hazard rate (elasticity) but the relative size of this reverse hazard rate "corrected" by the term $F(c) \max _{l \in\{1,2, \ldots, Q\}}\left\{v_{l}-v_{l+1}\right\}$ that also appears in the super-regularity condition. Again, the extra term reflects the relative size of the gambles in the different markets, given the aggregate uncertainty that the monopsonist faces. By strengthening the standard condi- 
tion on elasticity taking these gambles into account, we recover the "correct" ranking of prices.

Our one-seller-one-unit set-up can be easily extended to multiunit sellers, as long as they have constant marginal costs. While our procedure would allow the buyer to make a different price offer for each unit of a seller, optimally he would set a constant price for all. Increasing (decreasing) marginal costs would introduce the usual incentives towards distributing (concentrating) procurement over suppliers and would take us away from the classical model.

We have dealt with bilateral commitment in static games. In dynamic games and without dynamic (multilateral) commitment, the monopsonist could make their future decisions depend on past realizations of trade. That dynamic monopoly problem is an interesting extension of this paper.

The restriction to static mechanisms imposed by our main goal of microfounding monopsony, makes it impractical to think about our model in a mechanism design context: the sequential resolution of uncertainty would clearly be beneficial. Nonetheless, it is of note that our personalized pricing scheme is the best mechanism the buyer can devise subject to bilateral commitment in the static context.

Finally, it is important to point out that the personalized price setting mechanism that we analyze in this paper can be usefully adapted to the context of competing price setters. Burguet and Sákovics (2017a, 2017b, 2019) are witnesses to this. In the first paper, personalized pricing leads to a model of simultaneous price competition without the need for rationing (in case, given prices, demand exceeds supply) or demand sharing (in case, given prices, supply exceeds demand) as these are determined endogenously by the equilibrium bid vectors. The equilibrium is unique even when marginal cost are increasing: the price is competitive with positive profits.

In the second paper there is competition for input between two firms that also compete in the product market. Here, personalized pricing allows firms to strategically target their offers at the suppliers of their competitors. The "competitive foreclosure" that ensues leads to higher aggregate input (and, therefore output and efficiency), contrary to the 
usual foreclosure logic, which tends to lead to inefficiency.

The third paper extends the previous study to the case where the product market is collusive, as in the competition for talent in a sports league. It provides micro-foundations for some classical invariance theorems in the literature. 


\section{Appendix A: Third-degree price discrimination}

Aggregate uncertainty might affect the direction of third-degree price discrimination. Recall that, according to the classical multi-market monopsony model, ${ }^{20}$ the buyer should optimally offer a higher price to the market with the higher price elasticity of supply. This result need not hold in our model with uncertainty.

Indeed, let us reintroduce ex-ante (observable) asymmetry among sellers. To consider the simplest case, suppose there are two "markets" with $Q^{1}$ and $Q^{2}$ sellers, their cost distributions being $F($.$) and G($.$) , respectively. In order to calculate the expected marginal$ value we first need to calculate the probability that $l$ items are sold when the buyer offers $p^{1}$ to $Q^{1}$ sellers in market 1 and $p^{2}$ to $Q^{2}$ sellers in market 2 . First, let us denote by $\chi_{l}^{i}(x ; K)$ the value of $\chi_{l}(x)$ when $Q=K$ and the distribution function is the one characterizing suppliers in market $i$, for $i=1,2$. Also, and to save in notation, let $\chi_{l}^{i}(x ; K)=0$ whenever $l \geq K$. We should also introduce an additional piece of notation:

$$
\psi_{l}\left(p^{1}, p^{2} ; K^{1}, K^{2}\right)=\sum_{k=0}^{l} \chi_{k}^{1}\left(p^{1} ; K^{1}\right) \chi_{l-k}^{2}\left(p_{2} ; K^{2}\right)
$$

$\psi_{l}$ represents the probability that $l$ offers are accepted when $K^{i}$ are made to sellers in market $i$, each with a price of $p^{i}$. We can now write the corresponding system of equations for (4) as

$$
\begin{gathered}
\sum_{j=1}^{Q^{1}+Q^{2}} \psi_{j-1}\left(p^{1}, p^{2} ; Q^{1}-1, Q^{2}\right) v_{j}=\frac{F\left(p^{1}\right)}{f\left(p^{1}\right)}+p^{1} \\
\sum_{j=1}^{Q^{1}+Q^{2}} \psi_{j-1}\left(p^{1}, p^{2} ; Q^{1}, Q^{2}-1\right) v_{j}=\frac{G\left(p^{2}\right)}{g\left(p^{2}\right)}+p^{2} .
\end{gathered}
$$

It is now straightforward to generalize Proposition 1 to two (or more) classes of sellers.

Corollary 1 When both cost distributions are super-regular relative to the buyer's demand function, the optimal personalized pricing strategy is a posted price in each market, satisfying (5)-(6).

\footnotetext{
${ }^{20}$ See, for example, Tirole (1988) page 137.
} 
We are not particularly interested in the uniqueness of the pairs of (uniform) prices solving the first-order conditions. (In case there are several, the buyer simply chooses the pair maximizing his expected utility.)

Returning to third-degree price discrimination, note that in the solution to (5), the monopsonist again equals the marginal expenditure in market 1 (the right hand side) to the expected willingness to pay for the marginal unit, the left hand side. The subtle point here is that this expectation is taken conditional on all offers made in market 2 , and all but one made in market 1. Similarly, the solution to (6) depends on the same expectation but conditional on all but one offers in market 2 and all offers in market 1.

Note that, for $K^{1}+K^{2}-1 \geq l \geq 1$,

$$
\begin{aligned}
& \psi_{l}\left(p^{1}, p^{2} ; K^{1}, K^{2}\right) \\
= & \left(1-F\left(p^{1}\right)\right) \psi_{l}\left(p^{1}, p^{2} ; K^{1}-1, K^{2}\right)+F\left(p^{1}\right) \psi_{l-1}\left(p^{1}, p^{2} ; K^{1}-1, K^{2}\right) \\
= & \left(1-G\left(p^{2}\right)\right) \psi_{l}\left(p^{1}, p^{2} ; K^{1}, K^{2}-1\right)+G\left(p^{2}\right) \psi_{l-1}\left(p^{1}, p^{2} ; K^{1}, K^{2}-1\right) .
\end{aligned}
$$

Indeed, the second line above simply separates one supplier from the first market, computes the probability that $l$ units are obtained from the $K^{1}-1$ and $K^{2}$ other suppliers, and the probability that $l-1$ units are obtained from them. Then, it multiplies these probabilities by the probability of obtaining no unit or one unit from the separated supplier, respectively. The third line is a similar exercise with a separated supplier from market 2. Using (7), we can write the left-hand side of (5) as

$$
\begin{aligned}
& \sum_{j=1}^{Q^{1}+Q^{2}} \psi_{j-1}\left(p^{1}, p^{2} ; Q^{1}-1, Q^{2}\right) v_{j} \\
= & \left(1-G\left(p^{2}\right)\right) \sum_{j=1}^{Q^{1}+Q^{2}-1} \psi_{j-1}\left(p^{1}, p^{2} ; Q^{1}-1, Q^{2}-1\right) v_{j}+ \\
& G\left(p^{2}\right) \sum_{j=1}^{Q^{1}+Q^{2}-1} \psi_{j-1}\left(p^{1}, p^{2} ; Q^{1}-1, Q^{2}-1\right) v_{j+1} \\
= & \sum_{j=1}^{Q^{1}+Q^{2}-1} \psi_{j-1}\left(p^{1}, p^{2} ; Q^{1}-1, Q^{2}-1\right) v_{j} \\
& -G\left(p^{2}\right) \sum_{j=1}^{Q^{1}+Q^{2}-1} \psi_{j-1}\left(p^{1}, p^{2} ; Q^{1}-1, Q^{2}-1\right)\left(v_{j}-v_{j+1}\right),
\end{aligned}
$$


where we have also used the fact that

$$
\begin{aligned}
\psi_{0}\left(p^{1}, p^{2} ; Q^{1}-1, Q^{2}\right) & =\left(1-G\left(p^{2}\right)\right) \psi_{0}\left(p^{1}, p^{2} ; Q^{1}-1, Q^{2}-1\right), \\
\psi_{Q^{1}+Q^{2}-1}\left(p^{1}, p^{2} ; Q^{1}-1, Q^{2}\right) & =G\left(p^{2}\right) \psi_{Q^{1}+Q^{2}-2}\left(p^{1}, p^{2} ; Q^{1}-1, Q^{2}-1\right)
\end{aligned}
$$

Similarly for the left hand side of (6). Thus, we can write (5)-(6) as

$$
\begin{aligned}
& E_{p^{1}, p^{2}} v_{j}=\frac{F\left(p^{1}\right)}{f\left(p^{1}\right)}+p^{1}+G\left(p^{2}\right) E_{p^{1}, p^{2}} \Delta v_{j}, \\
& E_{p^{1}, p^{2}} v_{j}=\frac{G\left(p^{2}\right)}{g\left(p^{2}\right)}+p^{2}+F\left(p^{1}\right) E_{p^{1}, p^{2}} \Delta v_{j},
\end{aligned}
$$

where $E_{p^{1}, p^{2}} v_{j}=\sum_{j=1}^{Q^{1}+Q^{2}-1} \psi_{j-1}\left(p^{1}, p^{2} ; Q^{1}-1, Q^{2}-1\right) v_{j}$ is the expected (marginal) value of a unit bought from a supplier, given the offers made to $Q^{1}-1$ suppliers in market 1 and to $Q^{2}-1$ suppliers in market 2; and $E_{p^{1}, p^{2}} \Delta v_{j}$ is the expectation of the marginal increase in this value relative to the case where one more unit is bought from the other sellers, $\Delta v_{j}=v_{j}-v_{j+1}, E_{p^{1}, p^{2}} \sum_{j=1}^{Q^{1}+Q^{2}-1} \psi_{j-1}\left(p^{1}, p^{2} ; Q^{1}-1, Q^{2}-1\right)\left(v_{j}-v_{j+1}\right)$.

Now recall that the price elasticity of supply in market 1 is

$$
\varepsilon_{1}^{s}=\left(\frac{d F(p)}{d p} \frac{p}{F(p)}\right)^{-1}=\left(\frac{f(p)}{F(p)} p\right)^{-1} .
$$

Thus, the supply elasticity of a market at any price is proportional to the (inverse) hazard rate. The intuition becomes clear if we write the optimal pricing formula in terms of the hazard rate

$$
\begin{aligned}
& E_{p^{1}, p^{2}} v_{j}-p^{1}=\frac{F\left(p^{1}\right)}{f\left(p^{1}\right)}+G\left(p^{2}\right) E_{p^{1}, p^{2}} \Delta v_{j} \\
& E_{p^{1}, p^{2}} v_{j}-p^{2}=\frac{G\left(p^{2}\right)}{g\left(p^{2}\right)}+F\left(p^{1}\right) E_{p^{1}, p^{2}} \Delta v_{j} .
\end{aligned}
$$

The inverse (reverse) hazard rate may be higher, yet the optimal price in that market may be lower.

Note that, for all prices, $E_{p^{1}, p^{2}} \Delta v_{j} \leq \max _{j} \Delta v_{j}$. Thus, once again, aggregate uncertainty may results in changes that are related to the the additional term $F(x) \max _{j} \Delta v_{j}$. Combining the two equations in (8) we obtain

$$
\frac{G\left(p^{2}\right)}{g\left(p^{2}\right)}-\frac{F\left(p^{1}\right)}{f\left(p^{1}\right)}+\left(F\left(p^{1}\right)-G\left(p^{2}\right)\right) E_{p^{1}, p^{2}} \Delta v_{j}=p^{1}-p^{2} .
$$


Recall that reverse-hazard-rate dominance implies first order stochastic dominance. Then, the following proposition is immediate:

Proposition 4 Suppose that both $F$ and $G$ are super-regular relative to $\left\{v_{l}\right\}$, and for any $x \in(0,1), \frac{F(x)}{f(x)}<\frac{G(x)}{g(x)}$ (F reverse-hazard-rate dominates $G$ 's; i.e., market 1 's supply is more elastic than market 2 's). A sufficient condition for the optimal monopsony price in market 1 to be larger than that in market 2 is that for all $x$

$$
\frac{F(x)}{f(x)}-F(x) \max _{j} \Delta v_{j} \leq \frac{G(x)}{g(x)}-G(x) \max _{j} \Delta v_{j} .
$$

Thus, just as a strengthening of regularity guarantees that the posted prices are indeed optimal for a monopsonist, strengthening the inverse hazard rate dominance along the same lines guarantees that prices for a third-degree price discriminating monopsonist follow the same pattern as in the classical model of monopsony.

As an illustration that when the sufficient condition is not satisfied we can indeed obtain the "wrong" price ordering, consider the following example: ${ }^{21}$

Example 5 Let, $Q^{1}=Q^{2}=1 v_{1}=1$ and $v_{2}=0, F(x)=x$ and $G(x)=x^{2}$ if $x \leq .5$ and $G(x)=1.5 x-.5$ for $x>.5 .^{22}$ Then (5)-(6) become

$$
\begin{aligned}
& \left.\begin{array}{cc}
1-\left(p^{2}\right)^{2} & \text { if } \quad p^{2} \leq .5 \\
1.5\left(1-p^{2}\right) & \text { if } \quad p^{2}>.5
\end{array}\right\}=2 p^{1} \\
& 1-p^{1}=\left\{\begin{array}{c}
1.5 p^{2} \quad \text { if } \quad p^{2} \leq .5 \\
2 p^{2}-1 / 3 \quad \text { if } p^{2}>.5
\end{array}\right. \text {. }
\end{aligned}
$$

Solving, we obtain $p^{2}=\frac{3-\sqrt{5}}{2}<.5<p^{1}=\frac{\sqrt{5}}{2} \cdot \frac{3-\sqrt{5}}{2}$, while the price elasticities are $p^{1} \cdot \frac{f\left(p^{1}\right)}{F\left(p^{1}\right)} \equiv 1<p^{2} \cdot \frac{g\left(p^{2}\right)}{G\left(p^{2}\right)}=2$.

Once more, when the number of suppliers is large (and their capacity small, with respect to the size of the market), $\max _{j} \Delta v_{j}$ is small and (given regularity) the sufficient

\footnotetext{
${ }^{21}$ In fact, Example 4 could suffice, if we consider each seller as a different market, since elasticity is (constant and) equal in both markets in that case, yet prices are different.

${ }^{22}$ We cannot use $G(x)=x^{2}$ as it is not super-regular, so we could not appeal to the corollary.
} 
condition in Proposition 4 is satisfied. Consequently, the expected values in the left hand side of (5) and (6) for similar values of $p^{1}$ and $p^{2}$ approach, and then we recover the predictions of the classical monopsony model.

Finally, along the lines of Section 4, it is a straightforward corollary to Proposition 4 that the direction of third-degree price discrimination fixes itself when sellers are small:

Corollary 2 For $t$ sufficiently large, the market with higher price elasticity is offered the higher price.

\section{Appendix B}

\subsection{Proof of Lemma 1}

Suppose that it is optimal to offer a seller a price that is accepted for certain. As $\bar{c} \geq 1$, and the marginal value of this unit is bounded by $V(0)=1$, the maximum profit on this transaction is non positive. Then, as the probability that a price $p<1$ is accepted is $F(p)>0$, offering a lower price would lead to a positive profit. Consequently, offering a price above $\bar{c}$ can never be optimal.

Next, suppose that it is optimal to offer a price of 0 to a seller. Obviously, that would lead to no profit on that seller as the probability of acceptance is 0 . However, as long as there is a positive expected marginal valuation for that "last" unit, an offer to buy for a price above it will be accepted with positive probability - note that, given $\underline{c}=0$, there are always seller types below any positive value - and thus lead to positive marginal profit. The only way not to have a positive expected marginal valuation would be if the seller made at least one offer that is certainly accepted. However, we have just shown that such an offer is never made. Q.E.D. 


\subsection{Proof of Proposition 1}

By Lemma 1, we know that all prices are interior. We show that there can be no two different interior prices by reduction to the absurd. Take any two interior prices, $b^{j}, b^{k} \in$ $(0,1)$. Given the rest of the prices, we can compute the probabilities that the buyer obtains $l \in\{0,1, . ., Q-2\}$ units from these other sellers. Let those probabilities be denoted by $\tilde{\Phi}_{l}\left(b_{-(j, k)}\right)$. Then, the buyer's expected profit can be written as

$$
\sum_{l=0}^{Q-2} \tilde{\Phi}_{l}\left(b_{-(j, k)}\right)\left\{\left[F\left(b^{k}\right)+\left(1-F\left(b^{k}\right)\right) F\left(b^{j}\right)\right] v_{l+1}+F\left(b^{j}\right) F\left(b^{k}\right) v_{l+2}-F\left(b^{j}\right) b^{j}-F\left(b^{k}\right) b^{k}\right\} .
$$

To see this, note that, from the two sellers considered, the buyer will buy at least one unit if either he buys from the $k^{t h}$ seller (and either buys or not from the $j^{\text {th }}$ one) or if he does not buy from the $k^{\text {th }}$ but buys from the $j^{t h}$ seller. He will get a second unit if and only if he buys from both. Finally, he pays each seller if and only if he buys from them.

Thus, the first-order condition for $b^{j}$ is

$$
\sum_{l=0}^{Q-2} \tilde{\Phi}_{l}\left(b_{-(j, k)}\right)\left\{f\left(b^{j}\right)\left[F\left(b^{k}\right) v_{l+2}+\left(1-F\left(b^{k}\right)\right) v_{l+1}-b^{j}\right]-F\left(b^{j}\right)\right\}=0,
$$

and similarly for $b^{k}$. As $\sum_{l=0}^{Q-2} \tilde{\Phi}_{l}\left(b_{-(j, k)}\right)=1$, we can write this first-order condition in the familiar way (c.f. (3)),

$$
\frac{F\left(b^{j}\right)}{f\left(b^{j}\right)}+b^{j}=\widehat{v}^{j}
$$

where

$$
\widehat{v}^{j}=\sum_{l=0}^{Q-2} \tilde{\Phi}_{l}\left(b_{-(j, k)}\right)\left\{F\left(b^{k}\right) v_{l+2}+\left(1-F\left(b^{k}\right)\right) v_{l+1}\right\}
$$

is the expected value of the unit potentially bought from seller $j .{ }^{23}$ We can rewrite

$$
\begin{aligned}
\widehat{v}^{j} & =\sum_{l=0}^{Q-2} \tilde{\Phi}_{l}\left(b_{-(j, k)}\right) v_{l+1}-F\left(b^{k}\right) \sum_{l=0}^{Q-2} \tilde{\Phi}_{l}\left(b_{-(j, k)}\right)\left(v_{l+1}-v_{l+2}\right) \\
& =\sum_{l=0}^{Q-2} \tilde{\Phi}_{l}\left(b_{-(j, k)}\right) v_{l+1}-F\left(b^{k}\right) H\left(b_{-(j, k)}\right),
\end{aligned}
$$

\footnotetext{
${ }^{23}$ Note that, by regularity, the second-order condition is satisfied.
} 
where $H\left(b_{-(j, k)}\right)=\sum_{l=0}^{Q-2} \tilde{\Phi}_{l}\left(b_{-(j, k)}\right)\left(v_{l+1}-v_{l+2}\right)$. Then, the first-order conditions with respect to $b^{j}$ and $b^{k}$ imply

$$
\begin{aligned}
& \frac{F\left(b^{j}\right)}{f\left(b^{j}\right)}+b^{j}-F\left(b^{j}\right) H\left(b_{-(j, k)}\right)=\frac{F\left(b^{k}\right)}{f\left(b^{k}\right)}+b^{k}-F\left(b^{k}\right) H\left(b_{-(j, k)}\right)= \\
& \sum_{l=0}^{Q-2} \tilde{\Phi}_{l}\left(b_{-(j, k)}\right) v_{l+1}-\left[F\left(b^{k}\right)+F\left(b^{j}\right)\right] H\left(b_{-(j, k)}\right) .
\end{aligned}
$$

Therefore, if

$$
b+\frac{F(b)}{f(b)}-F(b) H\left(b_{-(j, k)}\right)
$$

is strictly monotone, we must have $b^{j}=b^{k}$. Finally, observe that, since $H\left(b_{-(j, k)}\right)=$ $\sum_{l=0}^{Q-2} \tilde{\Phi}_{l}\left(b_{-(j, k)}\right)\left(v_{l+1}-v_{l+2}\right) \leq \max _{l}\left\{v_{l}-v_{l+1}\right\}$, the strict monotonicity of $b+\frac{F(b)}{f(b)}-$ $F(b) \max _{l}\left\{v_{l}-v_{l+1}\right\}$ (and the monotonicity of $\left.F().\right)$ implies strict monotonicity of $(10)-$ and super-regularity implies the former. Repeating this argument for every pair of sellers, we obtain that all interior prices must be equal.

Finally, to obtain (4) just note that $\sum_{l=0}^{Q-2} \tilde{\Phi}_{l}\left(p^{D}\right)\left\{F\left(p^{D}\right) v_{l+2}+\left(1-F\left(p^{D}\right)\right) v_{l+1}\right\}=$ $\sum_{l=0}^{Q-1} \chi_{l}\left(p^{D}\right) v_{l+1}$. Q.E.D.

\subsection{Proof of Proposition 2}

Note that both prices are equating the virtual cost to: i) in the no uncertainty case, the demand function evaluated at the expected amount of trade; ii) in case of personalized pricing, the expected value of the marginal valuation (demand). Thus, as the virtual cost is strictly increasing by (super) regularity, all we need to check is whether, when $V$ is sufficiently concave,

$$
\sum_{l=0}^{Q-1} \chi_{l}(p) v_{l+1} \leq V(Q F(p))
$$

Note that

$$
\begin{aligned}
\sum_{l=0}^{Q-1} \chi_{l}(p) v_{l+1} & =\sum_{l=0}^{Q-1} \chi_{l}(p) \int_{l}^{l+1} V(x) d x=\sum_{l=0}^{Q-1} \int_{l}^{l+1} \chi_{l}(p) V(x) d x \\
& =\int_{0}^{Q} H(x ; p) V(x) d x
\end{aligned}
$$


where $H(x ; p)$ is the step (in $x)$ function $H(x ; p)=\chi_{l}(p)$ for all $x \in[l, l+1)$. Note that

$$
\int_{0}^{Q} H(x ; p) x d x=\sum_{l=0}^{Q-1} \chi_{l}(p) \int_{l}^{l+1} x d x=\sum_{l=0}^{Q-1} \chi_{l}(p)\left(l+\frac{1}{2}\right)=(Q-1) F(p)+\frac{1}{2} .
$$

Thus,

$$
\begin{aligned}
& V(Q F(p))-\sum_{l=0}^{Q-1} \chi_{l}(p) v_{l+1} \\
= & V(Q F(p))-V\left((Q-1) F(p)+\frac{1}{2}\right)+V\left(\int_{0}^{Q} H(x ; p) x d x\right)-\int_{0}^{Q} H(x ; p) V(x) d x .
\end{aligned}
$$

The first term is (positive if $F(p)>1 / 2$ and) related to the slope of $V$, whereas when $V$ is concave, the second is positive from Jensen's inequality. Also, the relative size of these terms depends on the degree of concavity of $V: V^{\prime \prime} / V^{\prime}$. Thus, when $V$ is sufficiently concave, the result follows. The result for (sufficient) convexity can be obtained in a similar way. Q.E.D.

\subsection{Proof of Lemma 2}

For each posted price $p$ and given the total number of sellers $t$, the number $j$ of sellers (other than $i$ ) that accept the offer, $n$ is a random variable with probability distribution, $\chi_{n}(p)$, a binomial with parameters $(t-1, F(c))$. Also, by the Strong Law of Large Numbers, these sellers' average supply converges a.s. to $F(p)$ as $t-1 \rightarrow \infty$. That is, taking into account that each seller sells $\delta=\frac{1}{t}$ when accepting the offer, and that $\frac{t-1}{t} \rightarrow 1$, total supply of these $t-1$ sellers converges a.s. to $F(c)$. That is,

$$
\operatorname{Pr}[|\delta n-F(c)|<\epsilon] \rightarrow 1, \quad \forall \epsilon>0
$$

Therefore, $\sum_{j / t \notin(F(p)-\epsilon, F(p)+\epsilon)} \chi_{j-1}(p) \rightarrow 0$ as $t \rightarrow \infty$, for all $\epsilon$, and the result follows. Q.E.D.

\subsection{Proof of Lemma 3}

That interior prices must be uniform for high enough $t$ follows immediately from the proof of Proposition 1. We only need to observe that, as $V($.$) is continuous, \max _{l}\left\{v_{l}-v_{l+1}\right\}$ 
converges to zero as $t \rightarrow \infty$, and so $H\left(b_{-(j, k)}\right)$ (an expectation of these values) does too. Consequently, regularity is sufficient for a unique interior solution.

To show that for high enough $t$ no extreme offer will be made, we will first prove that in equilibrium the marginal valuation must eventually be strictly above $\underline{c}$. It then follows that it is in the buyer's interest to make serious offers.

Take a price which is strictly above $\underline{c}$ (there must be at least one since $\underline{c}<1=v_{1}$ ). The marginal valuation for this unit must be at least as much as the price. Now take another price which is not serious. As $t$ increases, the difference between the marginal valuations of these two units converges to zero, so the second unit is also worth a serious price.

Next, note that unless the classical monopsony price equals $\bar{c}-$ which happens if the lowest marginal valuation is above the highest virtual cost - it must be the case that, for $t$ large enough, the marginal valuation is less than the highest virtual cost, implying that (4) has an interior solution. Q.E.D. 


\section{References}

[1] Akbarpour, M. and Li, S. (2018) "Credible mechanisms," in Proceedings of the 2018 ACM Conference on Economics and Computation, 371-371.

[2] Alonso, R., Matouschek, N. and W. Dessein (2008) "Centralization versus decentralization: An application to price setting by a multi-market firm," Journal of the European Economic Association, 6(2-3), 457-467.

[3] Bester, H. (1993) "Bargaining vs. price competition in markets with quality uncertainty," American Economic Review, 83, 278-288.

[4] Bulow, J. and J. Roberts (1989) "The simple economics of optimal auctions," Journal of Political Economy, 97(5), 1060-1090.

[5] Burguet, R. and J. Sákovics (1999) "Imperfect competition in auction designs," International Economic Review, 40(1), 231-247.

[6] Burguet, R. and J. Sákovics (2017a) "Bertrand and the long run," International Journal of Industrial Organization, 51, 39-55.

[7] Burguet, R. and J. Sákovics (2017b) "Competitive foreclosure," RAND Journal of Economics, 48(4), 906-926.

[8] Burguet, R. and J. Sákovics (2019) "Bidding for talent in sport," Economic Inquiry, $57(1), 85-102$.

[9] Chen, C.-H. and J. Ishida (2013) "Auctions versus negotiations: the role of price discrimination," ISER Discussion Paper No. 873, May 2013.

[10] Harris, M. and A. Raviv (1981) "A theory of monopoly pricing schemes with demand uncertainty," American Economic Review, 71(3), 347-365.

[11] Kotowski, M. (2018) "On Asymmetric Reserve Prices," Theoretical Economics, 13(1), $205-237$.

[12] Li, S. (2017) "Obviously strategy-proof mechanisms," American Economic Review, 107(11), 3257-87. 
[13] Martin, I. and R. Pindyck (2015) "Averting catastrophes: The strange economics of Scylla and Charybdis," American Economic Review, 105(10), 2947-2985.

[14] McElheran, K. (2014) "Delegation in Multi-Establishment Firms: Evidence from IT Purchasing," Journal of Economics 85 Management Strategy, 23(2), 225-258.

[15] Mookherjee, D. (2006) "Decentralization, Hierarchies, and Incentives: A Mechanism Design Perspective," Journal of Economic Literature, 44(2), 367-390.

[16] Myerson, R. (1981) "Optimal auction design," Mathematics of Operations Research, $6(1), 58-73$.

[17] Riley, J., and R. Zeckhauser (1983) "Optimal selling strategies: When to haggle, when to hold firm," Quarterly Journal of Economics, 98(2), 267-289.

[18] Thomas, C. (2011) "Too many products: Decentralized decision making in multinational firms," American Economic Journal: Microeconomics, 3(1), 280-306.

[19] Tirole, J. (1988) The theory of industrial organization, MIT Press.

[20] Wang, R. (1993) "Auctions versus posted-price selling," American Economic Review $83,838-851$.

[21] Wang, R. (1995) "Bargaining versus posted-price selling," European Economic Review $39,1747-1764$.

[22] Winter, E. (2004) "Incentives and discrimination," American Economic Review, 94(3), 764-773. 